\title{
A Preliminary Analysis of User's Body Impact on Signal Polarization in WBANs
}

\author{
Kenan Turbic ${ }^{1}$, Mariella Särestöniemi ${ }^{2}$, Matti Hämäläinen ${ }^{2}$, Timo Kumpuniemi ${ }^{2}$, Luis M. Correia ${ }^{1}$ \\ ${ }^{1}$ INESC-ID / IST, University of Lisbon, Lisbon, Portugal, \{kenan.turbic, luis.m.correia\}@tecnico.ulisboa.pt \\ ${ }^{2}$ Centre for Wireless Communications (CWC), University of Oulu, Oulu, Finland, \\ \{mariella.sarestoniemi, matti.hamalainen, timo.kumpuniemi\}@oulu.fi
}

\begin{abstract}
This paper analyses the impact of the human body on antenna radiation characteristics, with a focus on the polarization aspect. The effect of the body tissues on a wristworn ultra-wideband double loop antenna radiation characteristics is investigated at 3,4 and $5 \mathrm{GHz}$, based on numerical full-wave simulations complemented with a voxel model of a hand. Results show a strong influence of the body on the gain and polarization characteristics; the radiation in the direction towards the body is suppressed by $20 \mathrm{~dB}$ or more, and the antenna polarization changes from a linear to an elliptical one. By simulating an off-body communications scenario with the user walking at a fixed distance from the offbody antenna, up to $6.5 \mathrm{~dB}$ lower received power is obtained by using the wearable antenna radiation pattern simulated with the hand phantom, compared to the case when the antenna in free space.
\end{abstract}

Index Terms - Body Area Networks, Wearable Antennas, Polarization, Off-Body Channel, Ultra-Wideband.

\section{INTRODUCTION}

Wireless Body Area Networks (WBANs) have gained a great deal of attention during the recent years, with a variety of applications in healthcare and other areas being reported [1]. With the small size, light weight and energy efficient design of wearable devices, WBANs are suitable for continuous long-term monitoring of the psychophysiological parameters of a person, without limiting their mobility. Together with other sensors embedded within a smart environment, WBANs can collect important information and deliver it to a remote location, for further processing or decision making, e.g., by health professionals, in the case of medical applications.

WBANs rely on off-body communications to exchange information among each other, or to interact with external devices. Due to the high level of user mobility and dynamic motion of wearable antennas, propagation conditions in the off-body channel can significantly change over time, where the body shadowing and signal depolarization effects play important roles. While the former effect is associated with the Line-of-Sight (LoS) obstructions by the user's body, i.e., introducing losses up to $50 \mathrm{~dB}$ [2], the latter occurs due to the influence of body tissues on wearable antenna radiation characteristics, physical misalignment between antennas, and polarization-selective reflection, diffraction and scattering in the propagation environment [3]. These effects have a strong impact on system performance, hence a proper channel knowledge is extremely important from the transceiver design and implementation perspectives.

This paper investigates the impact of the user's body on wearable antennas' polarization characteristics. Based on full-wave numerical simulations in CST Microwave Studio [4], the radiation characteristics of a double loop ultrawideband (UWB) are analyzed at 3, 4 and $5 \mathrm{GHz}$, for an antenna in free space or worn on the wrist. The obtained antenna gain patterns are used in an off-body channel simulator [5], to investigate the effects on the channel. Since the polarization aspect of WBAN channels is typically neglected, with the available studies mostly focusing on signal excitation at a certain polarization [6],[7], the main novelty of this work is in the analysis of the depolarization effect and influence of user dynamics in off-body channels.

The rest of the paper is organized as follows. The following Section II describes the wearable antenna design and simulation, and introduces the off-body channel model. The antenna radiation characteristics are analyzed in Section III, Section IV presents dynamic off-body channel simulation results, and the paper is concluded in Section V.

\section{ANTENNA AND CHANNEL SIMULATION}

\section{A. Wearable antenna}

The wearable antenna considered in this study is a double-loop UWB antenna, designed for on- and off-body communications [8]. The antenna can operate over the full UWB frequency band $3.1 \mathrm{GHz}-10.6 \mathrm{GHz}$ [9], including the sub-bands considered by the IEEE 802.16.5 standard for WBANs [10]. For the purpose of this work, three frequencies are considered, i.e. $3 \mathrm{GHz}, 4 \mathrm{GHz}$ and $5 \mathrm{GHz}$, representing the boundaries for the low-band UWB signal [10].

The analysis of the antenna radiation characteristics is based on simulations in CST MicroWave Studio [4], which uses the finite integration technique (FIT) to solve computational electromagnetic (EM) problems [11]. The analysis is performed for an antenna in free space, and for this case it is placed on the left wrist of a person. The latter was done with the use of a hand phantom in CST, extracted from a biological voxel model Tom, which resolution is $0.5 \times 0.5 \times 0.5 \mathrm{~mm}^{3}$. This model represents a detailed structure of the human body with the internal organs and the 


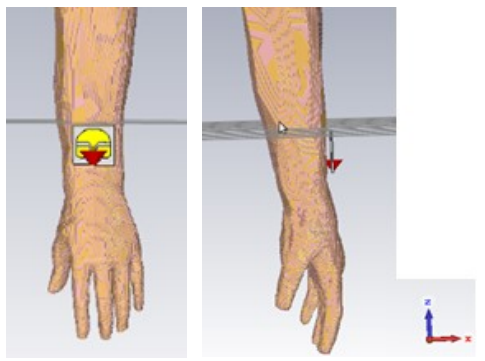

Fig. 1. Antenna location on the wrist of the voxel model.

corresponding frequency-dependent EM properties. The exact antenna placement on the phantom is shown in Fig. 1, where the antenna-body separation is set to $4 \mathrm{~mm}$, which provides reasonable antenna matching within practical antenna-body distance [12].

\section{B. Off-body channel with dynamic user}

The wearable antenna gain patterns obtained in free space and on the hand phantom (Section II-A) are used in an offbody channel simulator developed based on the polarized channel model presented in [5]. The model takes the antenna radiation characteristics, antennas' orientations, and the spatial configuration and EM properties of scatterers into account. It employs an analytical mobility model for wearable antennas on dynamic users based on Fourier series [13] to represent the influence of antenna displacement and rotation during motion. One should note that the channel model jointly considers the depolarization effect and the influence of user dynamics on the off-body channel.

In order to investigate the impact of the changed antenna polarization characteristics on the off-body channel, a scenario with the user walking in place at a $4 \mathrm{~m}$ distance from a $1.5 \mathrm{~m}$ high Access Point (AP) was considered. The received power is calculated for two orientations of the user, i.e., facing towards the AP, and facing sideways, with the AP on the user's left side (Fig. 2). The off-body antenna is at approximately $90^{\circ}$ azimuth angle relative to the wearable antenna's maximum radiation direction in the former case, while being aligned with it in the latter one.

In order to better understand the effects of wearable antenna polarization characteristics and signal depolarization, free space propagation is assumed herein, i.e. only the LoS component is present. Moreover, the off-body antenna is assumed to be an ideal vertically polarized isotropic radiator, so only on the effects associated with the wearable antenna are observed. The transmit power is set to

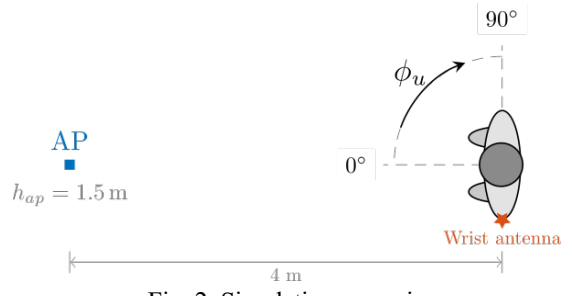

Fig. 2. Simulation scenario
$1 \mathrm{~W}(30 \mathrm{dBm})$ for simplicity, hence the received power directly corresponds to the channel transmission coefficient. Simulation was repeated for $3 \mathrm{GHz}, 4 \mathrm{GHz}$, and $5 \mathrm{GHz}$ carrier frequencies, with only one motion period being simulated in each case, due to the cyclic character of the walking motion.

\section{ANTENNA CHARACTERISTICS}

The radiation characteristics of the UWB double-loop antenna operating in free space and on the wrist phantom are shown in Fig. 3. As one observes for the free space case (Fig. 3a), the antenna pattern is omnidirectional in the $\theta$ polarization, with the gain being more than $20 \mathrm{~dB}$ higher than in the orthogonal polarization. On the other hand, the $\phi$ polarization gain is not uniform, having lobes at $k \cdot 45^{\circ}$ $(k=1,2,3,4)$, hence one expects the antenna polarization to slightly deviate from the vertical one in these directions.

As follows from Fig. 3b, placing the antenna on the body changes its radiation characteristics, with the most dominant effect observed in the back direction, i.e., towards the body. As previously reported in other studies, e.g. [2], the bodyshadowing loss in the backward direction exceeds $20 \mathrm{~dB}$, while increasing with frequency. Moreover, the number of minor lobes also increases with frequency, due to the interference between body-diffracted components, as their relative phases become more sensitive to the path length difference with electrically longer paths. It is also interesting to notice that the antenna gain in the direction perpendicular to the body is maximum at $5 \mathrm{GHz}$. This can be attributed to constructive interference between signal components reflected from the body, resulting in a better focus of the radiated power in this direction.

The difference between the $\theta$ - and $\phi$-polarization components is less drastic with antenna on the body, and the lower cross-polarization isolation (XPI) is observed. Therefore, in addition to shadowing losses, the user's body also affects the antenna's polarization. To gain more insight, Fig. 4 shows antenna polarization ellipses in a few selected azimuth directions; for the antenna in free space (Fig. 4 left column) and on the wrist (Fig. 4 right column). The ellipses are scaled according to the absolute gain in the corresponding direction, and normalized to the maximum value. Therefore, both the polarization and the relative field magnitude in the given direction are represented.

The antenna polarization in the free space case varies over azimuth directions, being linear and vertical at $0^{\circ}, 90^{\circ}$ and $180^{\circ}$, and elliptical at $45^{\circ}$ and $135^{\circ}$, with the axial ratio and major axis inclination varying with frequency.

The impact of the body on the radiation characteristics is evident in the case when antenna is attached to the wrist. Except for the direction away from the body (i.e., at $0^{\circ}$ ), the polarization is generally observed to change from a linear to an elliptical one, with frequency-variant properties. The most significant change is observed in the direction towards the body, with the polarization ellipse being almost horizontal in this case. Moreover, the corresponding ellipse is much smaller in size, due to the body-shadowing effects on the 


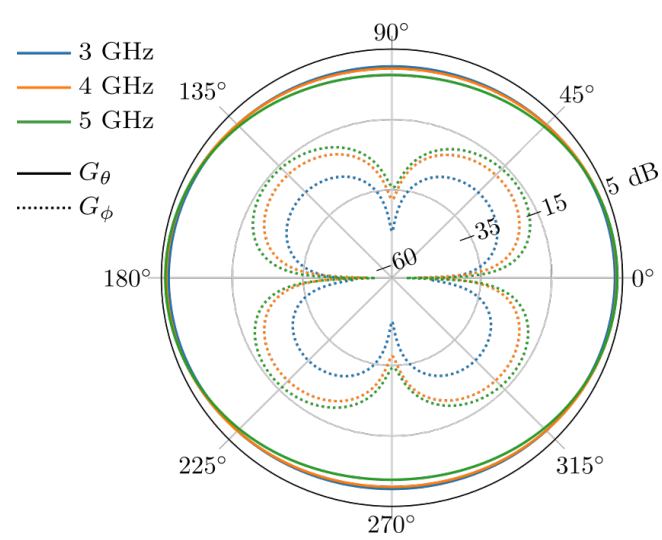

a) Antenna in free space

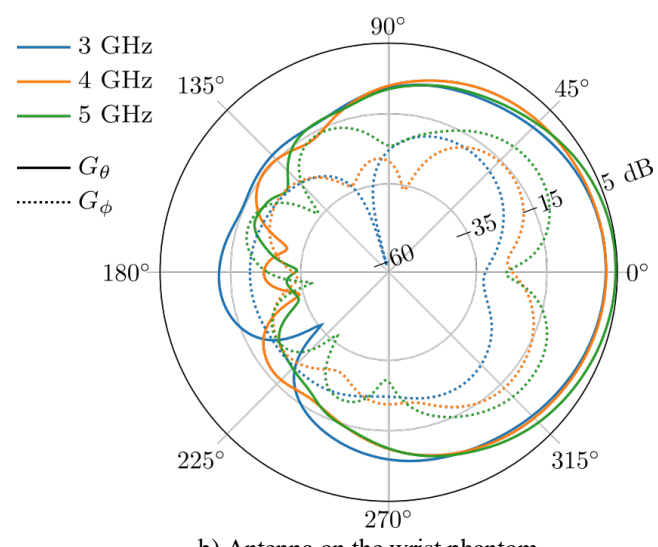

b) Antenna on the wrist phantom

Fig. 3. Antenna gain pattern in the azimuth plane.

field magnitude. On the other hand, the linear polarization is somewhat preserved in the forward radiation direction (i.e., $\left.0^{\circ}\right)$, although slightly deviating from the initial vertical one.

It is interesting to notice that the antenna polarization at $5 \mathrm{GHz}$ tends towards a linear one, for all considered azimuth angles except $45^{\circ}$. Albeit rotated from the vertical reference, i.e., as in the other cases, the polarization ellipses' eccentricities are the highest in this case.

\section{IMPACT ON THE OFF-BODY CHANNEL}

The received powers $\left(P_{r}\right)$ over a motion cycle obtained for the walking scenario (Section II-B) are presented in Fig. 5; for the user facing towards the AP antenna (Fig. 5a) and with their left side towards it (Fig. 5b). It is easy to verify from the figure that the received power variation is periodic with the same period as the user's motion, thereby justifying the choice to simulate only one motion cycle. One should note that the motion period $(T)$ is $1.14 \mathrm{~s}$ in the considered case, with the walking velocity being $1.31 \mathrm{~m} / \mathrm{s}$.

For the user facing towards the AP (Fig. 5a), there is a clear difference between the free-space case (solid line) and the one with antenna on the wrist phantom (dashed line). The former case shows higher $P_{r}$ values with low variation over the motion period, i.e., less than a $\mathrm{dB}$ at $3 \mathrm{GHz}$ and $4 \mathrm{GHz}$,
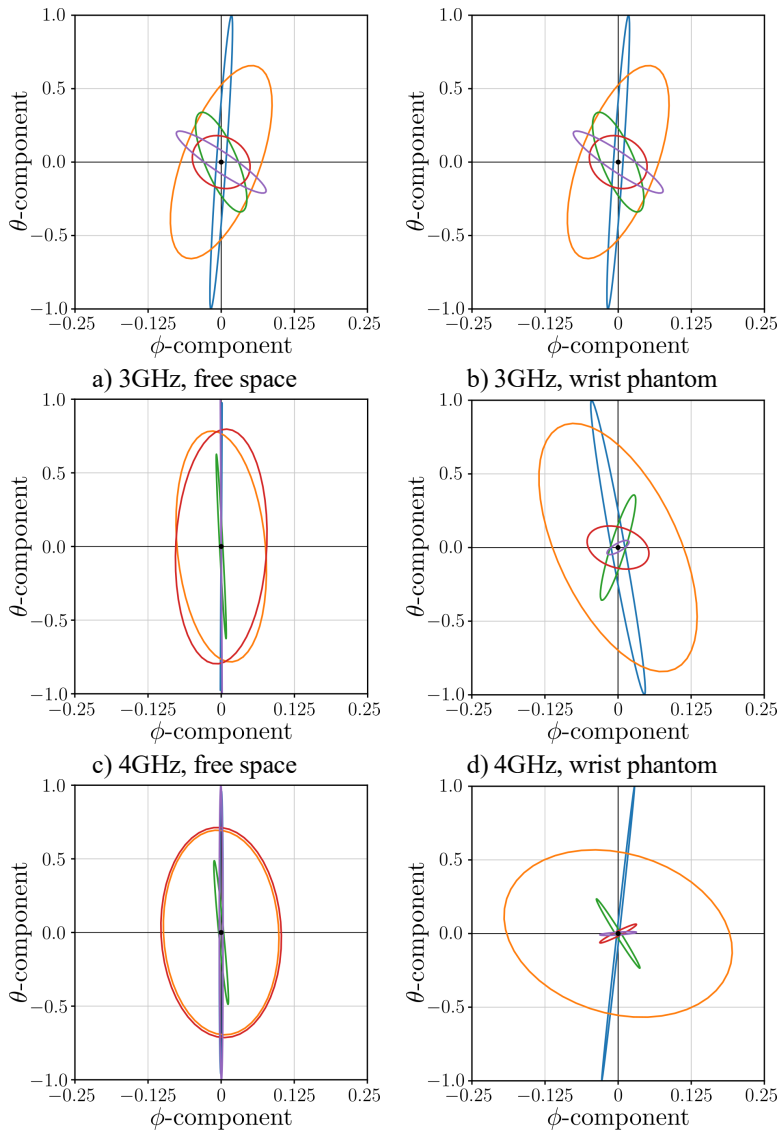

e) $5 \mathrm{GHz}$, free space

f) $5 \mathrm{GHz}$, wrist phantom

Fig. 4. Antenna polarization in the azimuth plane, for free space (left column) and with the wrist (right column).

while the latter one yields up to $6.5 \mathrm{~dB}$ lower levels and generally more dynamic signal variations. This difference is the result of changed antenna radiation characteristics near body tissue, with both absolute gain and XPI being affected.

The difference between the received power levels with antenna in free-space and on the wrist phantom is less significant in the case with the AP being in front of the wearable antenna (Fig. 5b). This agrees with the previous observations; the radiation characteristics of a body-worn antenna are least affected in the direction perpendicular to the skin. The signal variation in this case is attributed to variable gain and polarization losses associated with antenna rotation due to the swinging motion of the arm, with both effects having a similar contribution to the overall behavior.

One should note that the antenna in free space is observed to yield almost identical $P_{r}$ values at all frequencies, the difference being within $1 \mathrm{~dB}$. This shows that the antenna radiation characteristics around the $0^{\circ}$ azimuth are consistent over the considered frequency range. On the other hand, proximity of the body tissue introduces more frequency dependence, as observed from the wrist phantom case; $P_{r}$ can differ for more than $3 \mathrm{~dB}$ between $3 \mathrm{GHz}$ and $5 \mathrm{GHz}$. The highest $P_{r}$ is obtained at $5 \mathrm{GHz}$, as 


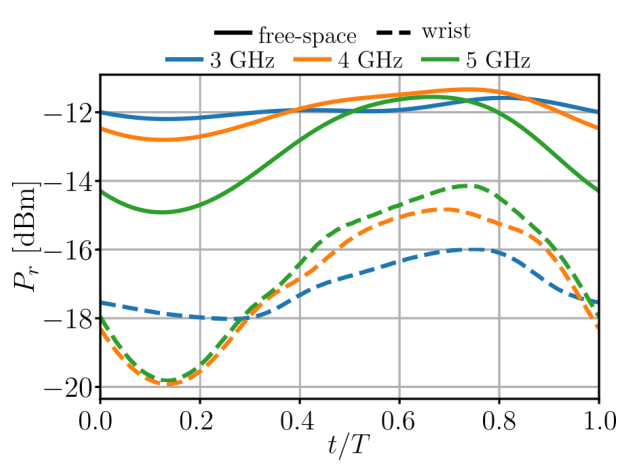

a) Antenna in free space

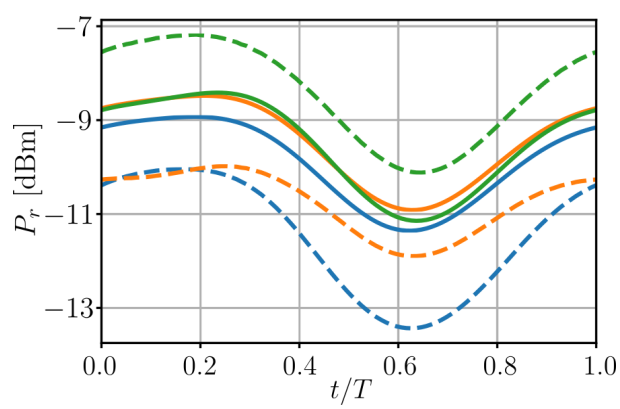

b) Antenna on the wrist phantom

Fig. 3. Antenna gain pattern in the azimuth plane.

the antenna is apparently better at focusing the radiated energy in the forward direction at this frequency, thereby yielding a slightly better performance (Fig. 5b).

\section{CONCLUSIONS}

With the increasing interest in WBAN applications, the need for improved energy efficiency, reliability and general system performance has driven research on wearable antennas and channel modeling. While the antenna detuning and body-shadowing effects are widely acknowledged effects in the presence of the body tissue, the ones on the antenna polarization are not adequately explored in literature. Moreover, the polarization aspect of the WBAN channels is typically neglected, although being recently reported to have an important effect on the receiver performance.

This paper considers the impact of the body on the radiation characteristics of a UWB double-loop antenna placed on the wrist, with a focus on the polarization characteristics. The antenna pattern simulated with a hand phantom was also used to simulate a free-space off-body communication scenario, with the user walking in place, with different orientations relative to the off-body antenna.

Results show a strong influence of the body on the antennas' radiation characteristics. In addition to the suppressed radiation in the back direction due to bodyshadowing, i.e., with the losses exceeding $20 \mathrm{~dB}$, the antenna polarization is generally observed to change from a linear to an elliptical one, where the major axis can be almost orthogonal to the principal polarization the antenna is designed for. The corresponding effects on the off-body channel with dynamic user include a change in the average received power, i.e., up to $6.5 \mathrm{~dB}$ in the considered scenario.

Future work will include a detailed analysis, considering additional wearable antenna placements and more dynamic user motions, e.g., running. Moreover, the off-body channel simulation results will be validated against anechoic chamber measurements.

\section{ACKNOWLEDGMENT}

The research at IST was performed within the framework of the COST Action CA15104, IRACON. The research at CWC has been financially supported partly by the project WBAN Communications in the Congested Environments (MeCCE) and in part by the Academy of Finland 6Genesis Flagship (grant 318927). Dr. Marko Sonkki is acknowledged for his advice with CST simulator.

\section{REFERENCES}

[1] P. S. Hall and Y. Hao, Antennas and Propagation for Body-Centric Wireless Communications, 2nd ed. Norwood, MA, USA: Artech House, 2012.

[2] S. L. Cotton, A. McKernan, A. J. Ali and W. G. Scanlon, "An Experimental Study on the Impact of Human Body Shadowing in Off-Body Communications Channels at 2.45 GHz," Proc. EuCAP2011, Rome, Italy, Apr. 2011.

[3] Y. He, X. Cheng, and G. L. Stüber, "On Polarization Channel Modeling,” IEEE Wireless Commun., vol. 23, no. 1, pp. 80-86, Feb. 2016.

[4] CST Microwave Studio, [Online]. Available: http://www.cst.com

[5] K. Turbic, L. M. Correia, and M. Beko, "A Channel Model for Polarised Off-Body Communications with Dynamic Users," IEEE Trans. Antennas Propag., 2019, In press.

[6] T. Tuovinen, M. Berg, E. Salonen, "Antenna Close to Tissue: Avoiding Radiation Pattern Minima With an Anisotropic Substrate," IEEE Antennas Wireless Propag. Lett., Vol.13, pp. 1680-1683, Aug. 2014.

[7] H. B. Lim, D. Baumann, J. Cai, R. Koh, E.P. Li, Y. Lu, "Antennae Polarization for Effective Transmission of UWB Signal around Human Body," Proc. ICUWB2007, Singapore, Sep. 2007.

[8] T. Tuovinen, K. Yekeh Yazdandoost, and J. Iinatti, "Comparison of the Performance of the two Different UWB Antennas for the Use in WBAN On-Body Communications," in Proc. EuCAP2012, Prague, Czech, Mar. 2012.

[9] The First Report and Order Regarding Ultra-Wideband Transmission Systems (2002) Federal Communication Commission, FCC 02-48, ET Docket No. 98-153, Washington D.C., USA, $94+$ appendices.

[10] IEEE Standard for Local and Metropolitan Area Networks, IEEE 802.15.6-2012 - Part 15.6: Wireless Body Area Networks, 2012.

[11] M. Särestöniemi, M. Hämäläinen, J. Iinatti, "An Overview of the Electromagnetic Simulation Based Channel Modeling Techniques for Wireless Body Area Network Applications”, IEEE Access - Special Section on Body Area Networks, Vol. 5, pp. 10622-10632, May 2017.

[12] M. Särestöniemi, C. Pomalaza-Raez, T. Kumpuniemi, M. Hämäläinen, J. Iinatti, "Measurement Data Based Study on the IntraBody Propagation in the Presence of the Sternotomy Wires and Aortic Valve Implant," IEEE Trans. Antennas Propag., Vol. 67, no. 8, pp. 4989-5001, Aug. 2019.

[13] K. Turbic, L. M. Correia, and M. Beko, "A Mobility Model for Wearable Antennas on Dynamic Users," IEEE Access, vol. 6, no. 1, pp. 63635-63648, Dec. 2018. 